\title{
On the velocities of flows consisting of cyclically monotone maps
}

\author{
A. Tudorascu
}

Follow this and additional works at: https://researchrepository.wvu.edu/faculty_publications

\section{Digital Commons Citation}

Tudorascu, A., "On the velocities of flows consisting of cyclically monotone maps" (2010). Faculty Scholarship. 267.

https://researchrepository.wvu.edu/faculty_publications/267 


\title{
On the Velocities of Flows Consisting of Cyclically Monotone Maps
}

\author{
A. TUdorascu
}

\begin{abstract}
Motivated by work on one-dimensional EulerPoisson systems, Gangbo et al. proved a surprisingly general flow-map formula which unequivocally links an absolutely continuous curve in the Wasserstein space to the corresponding family of optimal maps pushing forward a given reference measure to each measure on the curve. In this work we prove a similar result for higher dimensions. Possible applications to variational solutions for pressureless gas dynamics systems are discussed. These solutions are obtained as absolutely continuous curves in a new metric space which is topologically equivalent to the Wasserstein space.
\end{abstract}

\section{INTRODUCTION}

The classical theory of flows corresponding to maps $\mathbf{v}:[0, T] \times \mathbb{R}^{d} \rightarrow \mathbb{R}^{d}$ which are Lipschitz continuous in space uniformly with respect to time has become known as the Cauchy-Lipschitz theory. It basically shows (Picard-Lindelöf Theorem) that the solution $X(t ; \cdot)$ of

$$
\partial_{t} X(t ; \cdot)=\mathbf{v}(t, X(t ; \cdot)), X(0 ; \cdot)=\mathbf{i d}
$$

exists and it is unique for all times $t \in[0, T]$. For mere existence, the continuity of $\mathbf{v}$ is sufficient (Peano's Theorem). Uniqueness is also obtained under less stringent conditions such as one-sided Lipschitz condition or Osgood continuity [9]. If $\mathbf{v}$ is sufficiently regular, then $X(t ; \cdot)$ is a diffeomorphism of $\mathbb{R}^{d}$. More generally, if $\mathbf{v} \in C_{c}^{1}([0, T] \times \Omega)$, where $\Omega \subset \mathbb{R}^{d}$ is open, then the unique flow map $X(t ; \cdot)$ is a diffeomorphism of $\Omega$ [15], [16]. 
The flow equation above is closely related to the continuity equation from Fluid Dynamics

$$
\partial_{t} \mu+\nabla_{x} \cdot(\mu \mathbf{v})=0, \mu(0, \cdot)=\mu_{0} .
$$

Indeed, in a sufficiently smooth setting, the measures $\mu_{t}$ are given by

$$
\mu_{t}=X(t ; \cdot)_{\#} \mu_{0}
$$

i.e.,

$$
\int_{\mathbb{R}^{d}} \varphi \mathrm{d} \mu_{t}=\int_{\mathbb{R}^{d}} \varphi \circ X(t ; \cdot) \mathrm{d} \mu_{0} \quad \text { for all } t \in[0, T], \varphi \in C_{C}\left(\mathbb{R}^{d}\right) .
$$

The smooth setting becomes, however, unsatisfactory when one is looking at problems from mathematical physics, mainly systems of conservation laws (again, we recommend [9] for a concise outlook on this matter). The starting point is that a system of conservation laws can be thought of as a transport system in which $\mathbf{v}$ will depend on the actual density $\rho$. Due to formation of shocks (characteristics crossing), there is no "smooth" theory when it comes to linking the flow equation to the transport equation. To prove existence of essentially bounded solutions for the flow problem is easily achieved by smooth approximation even in the case of essentially bounded $\mathbf{v}$ with locally integrable distributional divergence. DiPerna and Lions published a celebrated paper [10] in which they developed a uniqueness theory based on the renormalization property of $\mathbf{v}$. It basically means that whenever $\rho$ is a weak solution for the transport equation, then so is $\phi(\rho)$ for smooth $\phi$. Obviously, classical solutions, if they existed, would satisfy that. Ambrosio [1] takes the question one step further and only assumes spatial $B V$ regularity of the vector field.

The problem we address here comes from the opposite perspective. We ask the question of whether given a family of Borel maps $X:[0, T] \times \Omega \rightarrow \mathbb{R}^{d}$ there exists a Borel velocity field $\mathbf{v}:[0, T] \times \mathbb{R}^{d} \rightarrow \mathbb{R}^{d}$ whose flow is $X(t ; \cdot)$. Of course, $X$ will have to be weakly differentiable in time for this question to even make sense. The motivation for our problem arose recently in joint work of the author with Gangbo and Nguyen [11], and the author with Nguyen [14]. It concerns hyperbolic-elliptic systems of partial differential equations of presureless Euler/Euler-Poisson type. More precisely [11] studies existence, uniqueness and regularity of variational solutions for the pressureless, repulsive Euler-Poisson system with constant background charge

$$
\begin{cases}\partial_{t} \rho+\partial_{x}(\rho v)=0 & \text { in }(0, T) \times \mathbb{R} \\ \partial_{t}(\rho v)+\partial_{x}\left(\rho v^{2}\right)=-\rho \partial_{x} \Phi & \text { in }(0, T) \times \mathbb{R} \\ -\partial_{x x}^{2} \Phi=\rho-1 & \text { in } \mathbb{R} .\end{cases}
$$


Among other things, in [14] nonvariational solutions were constructed for the pressureless Euler system

$$
\begin{cases}\partial_{t} \rho+\partial_{x}(\rho v)=0 & \text { in }(0, T) \times \mathbb{R}, \\ \partial_{t}(\rho v)+\partial_{x}\left(\rho v^{2}\right)=0 & \text { in }(0, T) \times \mathbb{R} .\end{cases}
$$

The velocity $v$ in either of the systems above is related to the optimal maps pushing the Lebesgue measure restricted to the unit interval forward to the current measure $\rho_{t}$ on the solution curve. The flow-map formula (1.4) from below, relating these optimal maps and $v$, is essential to proving that the weak solutions constructed in [11] for (1.2) are energy preserving. In [14], the same formula is the main ingredient for the argument that the sticky-particles solution for (1.3) obtained by Brenier and Grenier [6] does, indeed, satisfy the Oleinik entropy condition as it was conjectured in [6].

Let us recall some basic facts from the theory of $L^{2}$-absolutely continuous curves in $\mathcal{P}_{2}\left(\mathbb{R}^{d}\right)$ (the space of Borel probability measures on $\mathbb{R}^{d}$ with finite second-order moments). We shall be quite sketchy, for further details we recommend the comprehensive reference [3]. Let us endow $\mathcal{P}_{2}\left(\mathbb{R}^{d}\right)$ with the quadratic Wasserstein metric defined by

$$
W_{2}^{2}(\mu, v):=\inf _{\gamma} \iint_{\mathbb{R}^{d} \times \mathbb{R}^{d}}|x-y|^{2} \mathrm{~d} \gamma(x, y),
$$

where the infimum is taken among all probabilities $\gamma$ on the the product space $\mathbb{R}^{2 d}$ with marginals $\mu, v$. Thus, $\left(\mathcal{P}_{2}\left(\mathbb{R}^{d}\right), W_{2}\right)$ becomes a Polish space on which we define absolutely continuous curves by saying that $[0, T] \ni t \rightarrow \mu_{t} \in \mathcal{P}_{2}\left(\mathbb{R}^{d}\right)$ lies in $\operatorname{AC}^{2}\left(0, T ; \mathcal{P}_{2}\left(\mathbb{R}^{d}\right)\right)$ provided that there exists $f \in L^{2}(0, T)$ such that

$$
W_{2}\left(\mu_{t}, \mu_{t+h}\right) \leq \int_{t}^{t+h} f(s) \mathrm{d} s
$$

for all $0<t<t+h<T$. The metric derivative of such a curve is defined as

$$
\left|\mu^{\prime}\right|(t)=\lim _{s \rightarrow t} \frac{W_{2}\left(\mu_{s}, \mu_{t}\right)}{|s-t|} \text { for } \mathcal{L}^{1} \text {-almost every } t \in(0, T) .
$$

There exists a unique [3] Borel velocity field $\mathbf{v}:(0, T) \times \mathbb{R}^{d} \rightarrow \mathbb{R}^{d}$ such that $(\mu, \mathbf{v})$ satisfies (1.1) in the sense of distributions and $\left\|\mathbf{v}_{t}\right\|_{L^{2}\left(\mu_{t} ; \mathbb{R}^{d}\right)}=\left|\mu^{\prime}\right|(t)$ for almost every $t \in(0, T)$. This $\mathbf{v}$ is called the velocity of minimal norm associated to $\mu$, as it minimizes $\left\|\mathbf{w}_{t}\right\|_{L^{2}\left(\mu_{t} ; \mathbb{R}^{d}\right)}$ for almost every $t \in(0, T)$ among all Borel maps $\mathbf{w}:(0, T) \times \mathbb{R}^{d} \rightarrow \mathbb{R}^{d}$ that pair up with $\mu$ to satisfy (1.1). Furthermore, within the one-dimensional setting $d=1$, the following is true [17]: suppose $\mu, v \in \mathcal{P}_{2}(\mathbb{R})$ and let $M, N:\left(-\frac{1}{2}, \frac{1}{2}\right)=: I \rightarrow \mathbb{R}$ be the unique almost everywhere monotone 
nondecreasing maps such that $M_{\#} \mathcal{L}_{I}^{1}=\mu$ and $N_{\#} \mathcal{L}_{I}^{1}=v$, where $\mathcal{L}_{I}^{1}$ is the onedimensional Lebesgue measure restricted to $I$. Then $W_{2}(\mu, \nu)=\|M-N\|_{L^{2}(I)}$, and there is only one Borel velocity $v:(0, T) \times \mathbb{R} \rightarrow \mathbb{R}$ satisfying (1.1), so the minimality of the $L^{2}\left(\mu_{t}\right)$-norm as a selection principle is unnecessary here.

The following statement appears in [11].

Proposition 1.1. Suppose $\mu \in \mathrm{AC}^{2}\left(0, T ; \mathcal{P}_{2}(\mathbb{R})\right)$. Let $v$ be the velocity associated to $\mu$ and $M_{t}: I \rightarrow \mathbb{R}$ be monotone nondecreasing map such that $M_{t \#} \mathcal{L}_{I}^{1}=\mu_{t}$. For each $t$, modifying $M_{t}$ on a countable subset of I if necessary, we may assume without loss of generality that $M_{t}$ is left continuous. We have that

$$
v_{t}\left(M_{t} x\right)=\dot{M}_{t} x
$$

for $\mathcal{L}^{2}$-almost every $(t, x) \in(0, T) \times I$.

The main purpose of this paper is to present a similar result in higher dimensions.

The proof [11] of Proposition 1.1 is based on the observation that if $\mu \in$ $\mathrm{AC}^{2}\left(0, T ; \mathcal{P}_{2}(\mathbb{R})\right)$ and $M_{t}: I \rightarrow \mathbb{R}$ are as in the statement of the proposition, then

(1.5) For Lebesgue almost all $(t, x, y) \in(0, T) \times I^{2}, M_{t} x=M_{t} y$ implies $\dot{M}_{t} x=$ $\dot{M}_{t} y$

(in case both derivatives exist pointwise in some sense, later to be specified). Furthermore, the proof uses the fact that if $\mu \in \mathrm{AC}^{2}\left(0, T ; \mathcal{P}_{2}(\mathbb{R})\right)$ and $M_{t}: I \rightarrow \mathbb{R}$ is a monotone nondecreasing map such that $M_{t \#} \mathcal{L}_{I}^{1}=\mu_{t}$ for all $t \in[0, T]$, then $M \in H^{1}\left(0, T ; L^{2}(I)\right)$ and the metric derivative $\left|M^{\prime}\right|(t)$ exists at $t \in(0, T)$ if and only if the metric derivative $\left|\mu^{\prime}\right|(t)$ exists at $t$; in that case $\left|M^{\prime}\right|(t)=\left|\mu^{\prime}\right|(t)$. Note also that (1.5) is a necessary condition for (1.4) to hold.

Let us now return with a comment back to (1.1). Suppose $\varrho$ is an arbitrary Borel probability measure on $\mathbb{R}^{d}$. Note that (1.1) will still be satisfied by $\mu_{t}:=$ $X(t ; \cdot)_{\#} \varrho$ in the sense of distributions if $X \in H^{1}\left(0, T ; L^{2}\left(\varrho ; \mathbb{R}^{d}\right)\right)$ is such that $X_{t}:=X(t ; \cdot)$ is $\varrho$-essentially injective for $\mathcal{L}^{1}$-almost every $t \in(0, T)$. The velocity will be given by $v_{t}:=\dot{X}_{t} \circ X_{t}^{-1}$, a well defined map on the support of $\mu_{t}$ (which we assume to be Borel measurable in time-space). In this work we consider the case of $\varrho$ being the Lebesgue measure restricted to the unit cube of $\mathbb{R}^{d}$. The maps $M_{t}$ are cyclically monotone and for Lebesgue almost all $(t, y) \in(0, T) \times \mathbb{R}^{d}$ the fibers $X_{t}^{-1} y$ are either singletons or have positive $d$-dimensional Lebesgue measure. Thus, the essential injectivity assumption on $X$ is relaxed.

The next section formulates a generalization of Proposition 1.1 to multiple dimensions, the key assumption here being the multi-dimensional analogue of (1.5), i.e., (Eq). We show by means of a counterexample that, in dimensions higher than one, some extra conditions are needed in order for $(\mathrm{Eq})$ to hold. We provide two situations in which (Eq) does hold, namely $(\mathrm{H})$ (see Proposition 2.4) and the conditions in Proposition 2.5. 
In the last section we introduce a new metric space, topologically equivalent to the Wasserstein space $\mathcal{P}_{2}\left(\mathbb{R}^{d}\right)$. Within this framework, we provide sufficient conditions for variational solutions for the two-point boundary problem for the Euler-Monge-Ampère system to exist. We shall see that the essential injectivity of the maps $M_{t}$ is present here. Therefore, the generalization (Corollary 2.3) of Proposition 1.1 is unnecessary at this point. However, we will use this generalization to construct some special monokinetic solutions for the nonlinear Vlasov system with quadratic potential. Likewise, Corollary 2.3 will be applied to obtain velocities along geodesics in this space.

2. Curves of Cyclically Monotone Maps and Their Velocities Let $\Omega \subset \mathbb{R}^{d}$ be open. If $M \in H^{1}\left(0, T ; L^{2}\left(\Omega ; \mathbb{R}^{d}\right)\right)$, we denote by

$$
\dot{M} \in L^{2}\left(0, T ; L^{2}\left(\Omega ; \mathbb{R}^{d}\right)\right)
$$

its functional derivative. It is defined by

$$
\lim _{h \rightarrow 0}\left\|\frac{M_{t+h}-M_{t}}{h}-\dot{M}_{t}\right\|_{L^{2}\left(\Omega ; \mathbb{R}^{d}\right)}=0 \quad \text { for } \mathcal{L}^{1} \text {-almost every } t \in(0, T) .
$$

In the next lemma, we shall view $M$ as a map in $\operatorname{AC}^{2}\left(\mathbb{R} ; L^{2}\left(\Omega ; \mathbb{R}^{d}\right)\right)$ by extending $M_{t}=M_{0+}$ for $t \leq 0$ and $M_{t}=M_{T-}$ for $t \geq T$.

Lemma 2.1. Let $M \in H^{1}\left(0, T ; L^{2}\left(\Omega ; \mathbb{R}^{d}\right)\right)$ and $\dot{M}$ be its functional derivative. Then

$$
\lim _{h \rightarrow 0} \int_{0}^{T} \int_{\Omega}\left|\frac{M_{t+h} x-M_{t} x}{h}-\dot{M}_{t} x\right|^{2} \mathrm{~d} x \mathrm{~d} t=0 .
$$

As a consequence, there exist sequences $h_{k}^{+} \rightarrow 0^{+}, h_{k}^{-} \rightarrow 0^{-}$and a measurable subset $\mathcal{A} \subset \mathbb{R} \times \Omega$ such that $\left.\mathcal{L}^{2}(\mathbb{R} \times \Omega) \backslash \mathcal{A}\right)=0$ and

$$
\lim _{k \rightarrow \infty} \frac{M_{t+h_{k}^{+}} x-M_{t} x}{h_{k}^{+}}=\lim _{k \rightarrow \infty} \frac{M_{t+h_{k}^{-}} x-M_{t} x}{h_{k}^{-}}=\dot{M}_{t} x
$$

for all $(t, x) \in \mathcal{A}$.

The proof in [11] needs no modification. The philosophy behind this result is that, in some specified sense, $\dot{M}$ can be viewed as almost a classical pointwise time-derivative of $M$. We shall understand its importance in the sequel. Also, since $M \in H^{1}\left(0, T ; L^{2}\left(\Omega ; \mathbb{R}^{d}\right)\right)$, we have that it admits a Borel representative. Equation (2.1) shows that $\dot{M}$ itself has the property. Throughout the paper we identify both $M$ and $\dot{M}$ with their Borel representatives.

The analogue of (1.5) in the $d$-dimensional case is stated below.

(Eq) For Lebesgue almost all $(t, x, y) \in(0, T) \times \Omega \times \Omega, M_{t} x=M_{t} y$ implies $\dot{M}_{t} x=\dot{M}_{t} y$. 
The time derivative showing in this statement is in the sense of (2.2). Since the set $\mathcal{A}$ defined above has full measure, we have that $\dot{M}_{t} x, \dot{M}_{t} y$ both exist in that sense for almost all $(t, x, y) \in(0, T) \times \Omega \times \Omega$. Next we show that Proposition 1.1 can be extended to any family $M \in H^{1}\left(0, T ; L^{2}\left(\Omega ; \mathbb{R}^{d}\right)\right)$ provided that (Eq) holds. We begin with the following general result.

Theorem 2.2. Let $S, \tilde{S}:(0, T) \times \Omega \rightarrow \mathbb{R}^{d}$ be Borel maps. Then $S(t, x)=$ $S(t, y)$ implies $\tilde{S}(t, x)=\tilde{S}(t, y)$ for $\mathcal{L}^{2 d+1}$-almost every $(t, x, y) \in(0, T) \times \Omega \times \Omega$ if and only if there exists a Borel map $\mathbf{w}:(0, T) \times \mathbb{R}^{d} \rightarrow \mathbb{R}^{d}$ such that

$$
\mathbf{w}(t, S(t, x))=\tilde{S}(t, x)
$$

for $\mathcal{L}^{d+1}$-almost every $(t, x) \in(0, T) \times \Omega=: \Omega_{T}$.

Proof. The direct implication is obvious. We next prove the converse in two steps.

1. Let $\lambda$ denote the $d+1$-Lebesgue measure restricted to $(0, T) \times \Omega, \Phi$ : $(0, T) \times \Omega \rightarrow(0, T) \times \mathbb{R}^{d}$ given by $\Phi(t, x)=(t, S(t, x))$, and set $\vartheta:=\Phi_{\#} \lambda$. Denote by $\eta$ the vector-measure whose density with respect to $\lambda$ is $\tilde{S}$, then set $\sigma:=\Phi_{\#} \eta$. The components of $\sigma$ are the signed measures given by

$$
\begin{aligned}
& \sigma_{k}(B)=\iint_{\Phi^{-1}(B)} \tilde{S}_{k}(t, x) \mathrm{d} \lambda(t, x) \\
& \quad \text { for all Borel } B \subset(0, T) \times \mathbb{R}^{d}, k=1, \ldots, d .
\end{aligned}
$$

Their total variations satisfy $\left|\sigma_{k}\right| \ll \vartheta$ since $\mathcal{Y}(B)=\lambda\left(\Phi^{-1}(B)\right)$. Thus, we can apply the Radon-Nykodim theorem for signed measures to $\sigma_{k}$ and $\lambda$ for all $k=$ $1, \ldots, d$. We obtain a Borel vector field $\mathbf{w}:(0, T) \times \mathbb{R}^{d} \rightarrow \mathbb{R}^{d}$ such that $\mathrm{d} \sigma=$ $\mathbf{w} \mathrm{d} \boldsymbol{9}$.

2. We now apply the disintegration theorem (see, for example, Theorem 5.3.1 [3]) to the Borel vector field $\Phi$ and the measure $\lambda$. Thus, for 9 -almost every $(t, y) \in(0, T) \times \mathbb{R}^{d}$, there exists a unique Borel probability measure $\lambda_{t, y}$ on $\Omega_{T}$ such that the map $(t, y) \rightarrow \lambda_{t, y}(B)$ is Borel measurable for each Borel set $B \subset \Omega_{T}$. Furthermore, $\lambda_{t, y}\left(\Phi^{-1}(t, y)\right)=1$ for $\vartheta$-almost every $(t, y) \in(0, T) \times \mathbb{R}^{d}$ and

$$
\iint_{\Omega_{T}} f(t, x) \mathrm{d} \lambda(t, x)=\int_{0}^{T} \int_{\mathbb{R}^{d}} \int_{\Phi^{-1}(t, y)} f(t, x) \mathrm{d} \lambda_{t, y}(t, x) \mathrm{d} \mathcal{Y}(t, y)
$$

for every Borel measurable $f: \Omega_{T} \rightarrow[0, \infty]$. Take $f(t, x):=\varphi(t, M(t, x)) \tilde{S}(t, x)$ for an arbitrary Borel map $\varphi:(0, T) \times \mathbb{R}^{d} \rightarrow[0, \infty]$. We use the previous equation 
to infer

$$
\begin{aligned}
\iint_{\Omega_{T}} \varphi(t, S(t, x)) \tilde{S}(t, x) \mathrm{d} \lambda(t, x) \\
\quad=\int_{0}^{T} \int_{\mathbb{R}^{d}} \varphi(t, y) \int_{\Phi^{-1}(t, y)} \tilde{S}(t, x) \mathrm{d} \lambda_{t, y}(t, x) \mathrm{d} \vartheta(t, y) .
\end{aligned}
$$

But, according to step 1, we have that the integral in the left hand side above is equal to

$$
\int_{0}^{T} \int_{\mathbb{R}^{d}} \varphi(t, y) \mathbf{w}(t, y) \mathrm{d} \mathcal{Y}(t, y) .
$$

The arbitrariness of $\varphi$ yields

$$
\begin{aligned}
& \mathbf{w}(t, y)=\int_{\Phi^{-1}(t, y)} \tilde{S}(t, x) \mathrm{d} \lambda_{t, y}(t, x) \\
& \quad \text { for } \vartheta \text {-almost every }(t, y) \in(0, T) \times \mathbb{R}^{d} .
\end{aligned}
$$

Finally, since $\vartheta=\Phi_{\#} \lambda$, we can compute

$$
\begin{aligned}
\mathbf{w}(t, S(t, x))=\int_{\Phi^{-1}(t, S(t, x))} & \tilde{S}(t, z) \mathrm{d} \lambda_{t, S(t, x)}(t, z) \\
& \text { for } \lambda \text {-almost every }(t, x) \in \Omega_{T} .
\end{aligned}
$$

Note that $(t, z) \in \Phi^{-1}(t, S(t, x))$ is equivalent to $S(t, z)=S(t, x)$, so we can use the hypothesis to conclude.

The following result is a direct consequence of Theorem 2.2.

Corollary 2.3. Let $M \in H^{1}\left(0, T ; L^{2}\left(\Omega ; \mathbb{R}^{d}\right)\right)$. Then (Eq) holds if and only if there exists a Borel map $\mathbf{v}:(0, T) \times \mathbb{R}^{d} \rightarrow \mathbb{R}^{d}$ such that

$$
\mathbf{v}_{t}\left(M_{t} x\right)=\dot{M}_{t} x
$$

for $\mathcal{L}^{d+1}$-almost every $(t, x) \in(0, T) \times \Omega=: \Omega_{T}$.

Now we get back to (Eq). The example we give next shows that, unless extra conditions are imposed, this is exactly what is missing in higher dimensions.

Example. Let us consider $Q:=\left(-\frac{1}{2}, \frac{1}{2}\right)^{2}$ and the family of convex maps

$$
\Phi_{t}: Q \rightarrow \mathbb{R}, \quad \text { given by } \Phi_{t}\left(x_{1}, x_{2}\right)=\frac{x_{1}^{2}}{x_{2}+t+1}, t \in[0, T] .
$$

The spatial gradient

$$
M_{t} \mathbf{x}:=\nabla \Phi_{t}\left(x_{1}, x_{2}\right)=\left(\frac{2 x_{1}}{x_{2}+t+1},-\frac{x_{1}^{2}}{\left(x_{2}+t+1\right)^{2}}\right)
$$


lies in $H^{1}\left(0, T ; L^{2}\left(Q ; \mathbb{R}^{2}\right)\right)$ and is constant, for $t$ fixed, on any segment of the line $x_{1}=c\left(x_{2}+t+1\right)$ contained in $Q$. Indeed, for all

$$
c \in J:=\left[-\frac{1}{2 t+1}, \frac{1}{2 t+1}\right],
$$

the line $x_{1}=c\left(x_{2}+t+1\right)$ has a segment (degenerate only for the endpoints of $J$ ) contained in $Q$. It is worth noting that, in fact, as $C$ runs in $J$, these segments sweep the whole square $Q$ (see Figure 2.1 below for $t=0, c=1$ ). Obviously, $M_{t}$ is constantly $\left(2 c,-c^{2}\right)$ along such a segment. However, $\dot{M}_{t}$ takes different values at different points on any such segment, except on the portion of the $x_{2}$-axis contained in $Q$.

Indeed, for all

$$
c \in J:=\left[-\frac{1}{2 t+1}, \frac{1}{2 t+1}\right],
$$

the line $x_{1}=c\left(x_{2}+t+1\right)$ has a segment (degenerate only for the endpoints of $J$ ) contained in $Q$. It is worth noting that, in fact, as $c$ runs in $J$, these segments sweep the whole square $Q$ (see Figure 2.1 below for $t=0$, $c=1)$. Obviously, $M_{t}$ is constantly $\left(2 c,-c^{2}\right)$ along such a segment. However, $\dot{M}_{t}$ takes different values at different points on any such segment, except on the portion of the $x_{2}$-axis contained in $Q$.

On the left we show the graph of $\Phi_{t}$ for $t=0$. The intersection with the plane $x_{1}=x_{2}+1$ is a straight line segment over $Q$. Thus, the gradient of $\Phi_{0}$ is constant $(2,-1)$ along this segment.

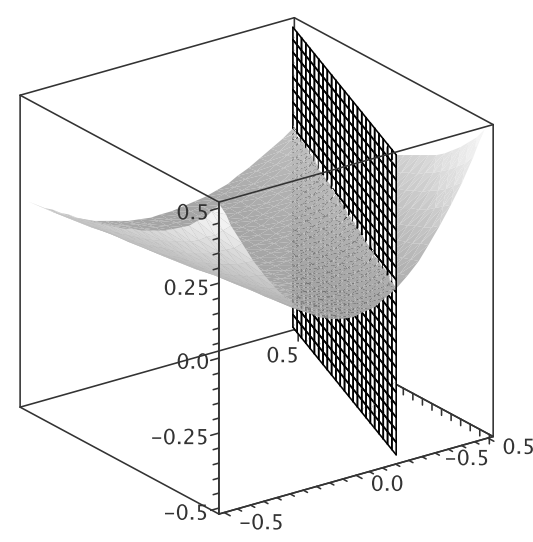

FIGURE 2.1. Graph of $\Phi_{0}$ and plane $x_{1}=$ $x_{2}+1$ over $Q$.

The example above shows that in higher dimensions we cannot expect a statement as general as Proposition 1.1 to hold, even if we drop the minimal velocity requirement on $\mathbf{v}$. The requirement $(\mathrm{Eq})$ is obviously satisfied if $M_{t}$ is invertible for almost every $t \in(0, T)$. We shall see, however, that the invertibility is, in fact, unnecessary and we shall give other necessary conditions for $(\mathrm{Eq})$ to hold.

In the sequel, $Q$ denotes the open unit cube in $\mathbb{R}^{d}$, centered at the origin. Furthermore, for all $t \in[0, T]$ the maps $M_{t}$ of the family $M \in H^{1}\left(0, T ; L^{2}\left(Q ; \mathbb{R}^{d}\right)\right)$ coincide $\mathcal{L}^{d}$-almost everywhere with the almost everywhere gradients of some convex functions $\Phi_{t}$ restricted to $Q$. For $(t, x) \in(0, T) \times Q=: Q_{T}$ define 
$\left[M_{t} x\right]:=\left\{y \in Q \mid M_{t} y=M_{t} x\right\}$. The following assumption will be used in the sequel:

(H) For almost every $(t, x) \in Q_{T},\left[M_{t} x\right]$ is either a singleton or $\mathcal{L}^{d}\left(\left[M_{t} x\right]\right)>$ 0 .

Due to the convexity of $\Phi_{t}$, it is easy to see that $\left[M_{t} x\right]$ is a convex set. Thus, if it does not consist of a single element, then $(\mathrm{H})$ implies that its interior is nonempty and convex.

We shall see that $(\mathrm{H})$ is sufficient for $(1.5)$ to hold. The example provided above, however, shows a case in which neither $(\mathrm{H})$ nor $(1.5)$ hold.

Proposition 2.4. Let $M \in H^{1}\left(0, T ; L^{2}\left(Q ; \mathbb{R}^{d}\right)\right)$ be such that for $\mathcal{L}^{1}$-almost every $t \in(0, T)$ the map $M_{t}$ coincides $\mathcal{L}^{d}$-almost everywhere with the restriction to $Q$ of a gradient of a convex function defined on $\mathbb{R}^{d}$. Then $(\mathrm{H})$ implies $(\mathrm{Eq})$.

Proof. According to Lemma 2.1, for almost every $t \in(0, T)(2.2)$ holds for almost every $x \in Q$. Consider $t_{0} \in(0, T)$ and $x_{0} \in Q$ such that $\left[M_{t_{0}} x_{0}\right]$ contains more than just $x_{0}$ and such that $\dot{M}_{t_{0}} x_{0}$ exists in the (2.2) sense. According to $(\mathrm{H})$, we may assume $x_{0} \in \operatorname{Int}\left[M_{t_{0}} x_{0}\right]$. Since $\operatorname{Int}\left[M_{t_{0}} x_{0}\right]$ is an open, convex set, we may choose $x_{1}, x_{2}, \ldots, x_{d} \in\left[M_{t_{0}} x_{0}\right]$ such that the directions $x_{0}-x_{i}$, $i=1, \ldots, d$ are linearly independent and all $\dot{M}_{t_{0}} x_{i}$ exist in the (2.2) sense. Let $\varphi(t)=\left(x_{0}-x_{1}\right) \cdot\left(M_{t} x_{0}-M_{t} x_{1}\right)$ defined on $(0, T)$. Clearly, $\varphi \in H^{1}(0, T)$. Since $M_{t}$ is, in particular, a monotone operator, we have $\varphi \geq 0$ in $(0, T)$. But $\varphi\left(t_{0}\right)=0$ and, since the pointwise derivative (in the (2.2) sense) $\varphi^{\prime}\left(t_{0}\right)=\left(\dot{M}_{t_{0}} x_{0}-\dot{M}_{t_{0}} x_{1}\right)$. $\left(x_{0}-x_{1}\right)$ exists at $t_{0}$ we infer that $\left(x_{0}-x_{1}\right) \cdot\left(y_{0}-y_{1}\right)=0$, where $y_{i}:=\dot{M}_{t_{0}} x_{i}$ for all $i=0,1, \ldots, d$. Likewise, we obtain

$$
\left(x_{i}-x_{j}\right) \cdot\left(y_{i}-y_{j}\right)=0 \text { for all } i, j=1, \ldots, d .
$$

Furthermore, due to the cyclical monotonicity of gradients of convex functions, we apply a similar argument to the time-variable function

$$
(0, T) \ni t \rightarrow\left(x_{i}-x_{j}\right) \cdot M_{t} x_{j}+\left(x_{j}-x_{k}\right) \cdot M_{t} x_{k}+\left(x_{k}-x_{i}\right) \cdot M_{t} x_{i} \leq 0
$$

which attains its maximum at $t_{0}$ (point of differentiability in the (2.2) sense) to obtain

$$
\left(x_{i}-x_{j}\right) \cdot y_{j}+\left(x_{j}-x_{k}\right) \cdot y_{k}+\left(x_{k}-x_{i}\right) \cdot y_{i}=0 \text { for all } i, j, k=1, \ldots, d \text {. }
$$

If we use (2.6) for the index pair $(j, k)$ and combine the equality obtained with (2.7) we deduce that $\left(y_{i}-y_{j}\right) \cdot\left(x_{i}-x_{k}\right)=0$. Since this holds for arbitrary indices, we infer, in particular, that $y_{0}-y_{1}$ is orthogonal to $x_{0}-x_{i}$ for all $i=1, \ldots, d$. Thus, $\dot{M}_{t_{0}} x_{0}=\dot{M}_{t_{0}} x_{1}$.

Example. If $d=1$, then $(\mathrm{H})$ is automatically satisfied [11]. The reason is that a "flat" portion in the graph of $M_{t}$ is necessarily of positive Lebesgue measure. 


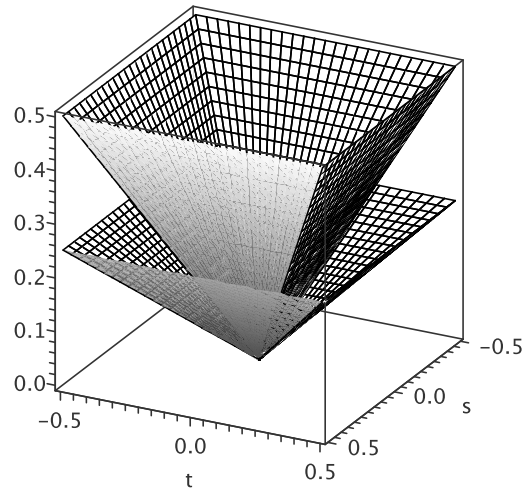

FIGURE 2.2. Graphs (parametric in $t, s)$ of $\Phi_{0.5}$ (below) and $\Phi_{1}$ over $Q$
We next give an example of a map $M$ satisfying $(\mathrm{H})$ in the case $d=2$. We construct explicitly the map $\mathbf{v}$.

Example. Take $d=2$ and consider the diagonals $d^{+}$: $x_{1}=x_{2}$ and $d^{-}: x_{1}=-x_{2}$ of the unit square $Q$ centered at $(0,0)$. They divide $Q$ into four subdomains, the closures of which are denoted by $D_{1}$ $\left(x_{1} \geq 0\right), D_{2}\left(x_{2} \geq 0\right), D_{3}$ $\left(x_{1} \leq 0\right), D_{4}\left(x_{2} \leq 0\right)$. Likewise, the bisectors $\left\{x_{1}=x_{2}\right\}$ and $\left\{x_{1}=-x_{2}\right\}$ divide the plane into the corresponding four closed regions denoted $\mathcal{D}_{i}$, $i=1, \ldots, 4$. Consider the family of convex maps $\left\{\Phi_{t}\right\}_{t \in[0,1]}$ given by

$$
\Phi_{t}(\mathbf{x})=\left\{\begin{array}{ll}
t x_{1} & \text { in } D_{1}, \\
t x_{2} & \text { in } D_{2}, \\
-t x_{1} & \text { in } D_{3}, \\
-t x_{2} & \text { in } D_{4},
\end{array} \text {, so that } M_{t} \mathbf{x}= \begin{cases}t \mathbf{e}_{1} & \text { in Int } D_{1}, \\
t \mathbf{e}_{2} & \text { in Int } D_{2}, \\
-t \mathbf{e}_{1} & \text { in Int } D_{3}, \\
-t \mathbf{e}_{2} & \text { in Int } D_{4} .\end{cases}\right.
$$

and

$$
\begin{aligned}
& M_{t} \mathbf{x}= \begin{cases}t\left(\mathbf{e}_{1}+\mathbf{e}_{2}\right) / 2 & \text { on }\left(D_{1} \cap D_{2}\right) \backslash\{(0,0)\}, \\
t\left(-\mathbf{e}_{1}+\mathbf{e}_{2}\right) / 2 & \text { on }\left(D_{2} \cap D_{3}\right) \backslash\{(0,0)\}, \\
-t\left(\mathbf{e}_{1}+\mathbf{e}_{2}\right) / 2 & \text { on }\left(D_{3} \cap D_{4}\right) \backslash\{(0,0)\}, \\
t\left(\mathbf{e}_{1}-\mathbf{e}_{2}\right) / 2 & \text { on }\left(D_{4} \cap D_{1}\right) \backslash\{(0,0)\}, \\
(0,0) & \text { if } \mathbf{x}=(0,0),\end{cases} \\
& N_{t}= \begin{cases}\mathbf{e}_{1} / 4 & \text { in Int } \mathcal{D}_{1}, \\
\mathbf{e}_{2} / 4 & \text { in Int } \mathcal{D}_{2}, \\
-\mathbf{e}_{1} / 4 & \text { in Int } \mathcal{D}_{3}, \\
-\mathbf{e}_{2} / 4 & \text { in Int } \mathcal{D}_{4}, \\
(0,0) & \text { elsewhere. }\end{cases}
\end{aligned}
$$


It is easy to see that here $\mathbf{v}_{t}:=\dot{M}_{t} \circ N_{t}$ does the job. Observe that we can throw out the diagonals $d^{ \pm}$to see that $(\mathrm{H})$ holds since $M_{t}$ is constant in the interior of each $D_{i}$.

We next discuss a situation, important from the optimal transportation point of view, where $(\mathrm{H})$ is not required for (1.5) to hold.

Proposition 2.5. For all integers $n \geq 0$ let $\Phi_{n}: \mathbb{R}^{d} \rightarrow \mathbb{R}$ be convex functions. Let $\alpha_{n}:(0, T) \rightarrow \mathbb{R}$ be Borel measurable and such that for each $n$ it is either positive on $(0, T)$ or identically null. Assume that for all $t \in(0, T)$ we have

$$
\sum_{n=1}^{\infty} \alpha_{n}(t) \Phi_{n}=\Phi_{t} \quad \text { almost everywhere in } Q
$$

and

$$
\sum_{n=1}^{\infty} \alpha_{n}(t) \nabla \Phi_{n}=M_{t} \quad \text { almost everywhere in } Q .
$$

Then for almost all $(t, x, y) \in(0, T) \times Q \times Q$ we have that $\nabla \Phi_{t}(x)=\nabla \Phi_{t}(y)$ implies $\nabla \Phi_{n}(x)=\nabla \Phi_{n}(y)$ for all $n$ for which $\alpha_{n}>0$ on $(0, T)$.

Proof. Let $t \in(0, T)$ be arbitrarily fixed. Clearly, $\Phi_{t}$ is convex (pointwise limit of convex functions). Set $M_{n}:=\nabla \Phi_{n}$. Let $\mathcal{G}_{t}$ be the subset of $Q$ of full measure where all gradients $\nabla \Phi_{n}, \nabla \Phi_{t}$ exist and where the convergence expressed in (2.8) and (2.9) takes place. Take an arbitrary $w \in G_{t}$. We use

$$
\Phi_{n}(z)-\Phi_{n}(w) \geq M_{n} w \cdot(z-w) \text { for all } n \geq 1, z \in G_{t}
$$

to conclude (using first partial sums and then taking the limit) that

$$
\Phi_{t}(z)-\Phi_{t}(w) \geq M_{t} w \cdot(z-w) \quad \text { for all } z \in G_{t} .
$$

Since $\mathcal{L}^{d}\left(Q \backslash G_{t}\right)=0$ and also $w \in G_{t}$, we infer $M_{t} w=\nabla \Phi_{t}(w)$. As $w$ was arbitrarily chosen in $G_{t}$, we deduce $M_{t}=\nabla \Phi_{t}$ everywhere in $G_{t}$. Now assume there exist $t \in(0, T)$ and $x, y \in G_{t}$ such that $M_{t} x=M_{t} y$. Part of the following argument is repetitive, but we need to record the inequalities for further reference. We write

$$
\Phi_{n}(x)-\Phi_{n}(y) \geq M_{n} y \cdot(x-y) \quad \text { for all } n \geq 1
$$

to conclude, as before, that

$$
\Phi_{t}(x)-\Phi_{t}(y) \geq M_{t} y \cdot(x-y) .
$$

Similarly,

$$
\Phi_{n}(y)-\Phi_{n}(x) \geq M_{n} x \cdot(y-x)
$$


implies

$$
\Phi_{t}(y)-\Phi_{t}(x) \geq M_{t} x \cdot(y-x) .
$$

Since their sum is an equality, we deduce that we must have equality in both (2.11) and (2.13). Consequently, we must have equality in (2.10) and (2.12) as well whenever $\alpha_{n}>0$, i.e.,

$$
\Phi_{n}(y)-\Phi_{n}(x)=M_{n} x \cdot(y-x)=M_{n} y \cdot(y-x)
$$

for all $n$ for which $\alpha_{n}>0$, and, since

$$
\Phi_{n}(z)-\Phi_{n}(y) \geq M_{n} y \cdot(z-y) \quad \text { for all } z \in Q,
$$

we get, by addition,

$$
\Phi_{n}(z)-\Phi_{n}(x) \geq M_{n} y \cdot(z-x) \quad \text { for all } z \in Q .
$$

We know $\Phi_{n}$ is differentiable at $x$, therefore, $M_{n} y=\nabla \Phi_{n}(x)=M_{n} x$ for all $n$ for which $\alpha_{n}>0$ on $(0, T)$.

Corollary 2.6. Let $\Phi_{0}, \Phi_{1}: \mathbb{R}^{d} \rightarrow \mathbb{R}$ be two convex functions and denote by $M_{0}, M_{1}$ the restrictions to $Q$ of their almost everywhere gradients. Consider the maps $M_{t}=(1-t) M_{0}+t M_{1}, t \in[0,1]$ and assume that $M_{t} x=M_{t} y$ for some $(t, x, y) \in$ $(0,1) \times\left[D\left(\Phi_{0}\right) \cap D\left(\Phi_{1}\right)\right]^{2}$, where $D(\Phi):=\{x \in Q \mid \Phi$ is differentiable at $x\}$. Then $\dot{M}_{t} x=\dot{M}_{t} y$.

Remark 2.7. In fact, we have just proved a stronger result than $\dot{M}_{t} x=\dot{M}_{t} y$. It is not surprising that the graph of the convex interpolation between $\Phi_{0}$ and $\Phi_{1}$ contains only those horizontal line segments which lie in the graphs of both $\Phi_{0}$ and $\Phi_{1}$.

Remark 2.8. Let $\mathcal{B}\left(Q ; \mathbb{R}^{d}\right)$ denote the set of Borel functions from $Q$ into $\mathbb{R}^{d}$. Note that an analytic family $\Phi \in C^{\omega}\left(0, T ; \mathcal{B}\left(Q ; \mathbb{R}^{d}\right)\right)$ with the property that

$$
\left.\frac{\partial^{n} \Phi}{\partial t^{n}}\right|_{t=0} \text { is convex for all } n \geq 0
$$

will, according to Proposition 2.6, satisfy (Eq). Consider the following example: $\Phi_{n}$ are convex functions satisfying $\left|\nabla \Phi_{n}(x)\right| \leq|\beta(x)|^{n}$ almost everywhere in $Q$ for all $n$ and some $\beta \in \mathcal{B}(Q ; \mathbb{R})$. Choose the $\Phi_{n}$ 's such that $\left|\Phi_{n}(x)\right| \leq$ $2\left|\nabla \Phi_{n}(x)\right|$ for all $n$ (see the proof of Proposition $3.2(\mathrm{i})$ ). Then,

$$
\Phi_{t}(x)=\sum_{n=0}^{\infty} \frac{t^{n}}{n !} \Phi_{n}(x) \text { and } \nabla \Phi_{t}(x)=\sum_{n=0}^{\infty} \frac{t^{n}}{n !} \nabla \Phi_{n}(x)
$$

satisfy the hypothesis of Proposition 2.6. 


\section{Applications to Pressureless Gas Dynamics}

3.1. Geodesiscs in the Wasserstein space. Let $T>0$ and $\rho_{0}, \rho_{T}$ be Borel probabilities with finite second moments such that Monge's problem of optimally transporting $\rho_{0}$ into $\rho_{T}$ has a solution. Brenier [5] showed that is equivalent to the existence of a gradient $M$ of a convex function such that $M_{\#} \rho_{0}=\rho_{1}$. It is well known (see McCann's interpolation [13]) that the geodesic in the Wasserstein space $\mathcal{P}_{2}\left(\mathbb{R}^{d}\right)$ connecting $\rho_{0}$ and $\rho_{T}$ is given by the formula

$$
[0, T] \ni t \rightarrow \rho_{t}:=[(1-t / T) \mathrm{Id}+(t / T) M]_{\#} \rho_{0} .
$$

Since $M_{t}:=(1-t / T) \operatorname{Id}+(t / T) M$ is invertible, we let $\mathbf{v}_{t}:=(1 / T)(M-\mathrm{Id}) \circ M_{t}^{-1}$ to see that $\dot{M}_{t}=\mathbf{v}_{t} \circ M_{t}$ for $t \in(0, T)$. Consider a smooth test function $\phi(t, y)$ such that $\phi(0, \cdot) \equiv 0 \equiv \phi(T, \cdot)$. Since $M \in H^{1}\left(0, T ; L^{2}\left(\rho_{0} ; \mathbb{R}^{d}\right)\right)$, we have that

$$
\int_{0}^{T} \int_{\mathbb{R}^{d}} \frac{\mathrm{d}}{\mathrm{d} t}\left[\phi\left(t, M_{t} x\right)\right] \mathrm{d} \rho_{0}(x) \mathrm{d} t=0
$$

due to the boundary conditions on $\phi$. The chain rule yields

$$
\int_{0}^{T} \int_{\mathbb{R}^{d}}\left\{\partial_{t} \phi\left(t, M_{t} x\right)+\dot{M}_{t} x \cdot \nabla_{y} \phi\left(t, M_{t} x\right)\right\} \mathrm{d} \rho_{0}(x) \mathrm{d} t=0,
$$

which, in view of $\dot{M}_{t}=\mathbf{v}_{t} \circ M_{t}$ and $\rho_{t}=M_{t \#} \rho_{0}$, gives the distributional form of the continuity equation from Fluid Mechanics

$$
\partial_{t} \rho+\nabla \cdot(\rho \mathbf{v})=0 .
$$

To obtain the momentum equation, note that $\ddot{M}_{t} \equiv 0$. Then we multiply this simple identity by $\phi\left(t, M_{t} x\right)$ and integrate in $x$ with respect to $\rho_{0}$. After that, we integrate by parts in time and take again into account the properties already used for getting (3.1), to obtain the distributional form of the momentum equation

$$
\partial_{t}(\rho \mathbf{v})+\nabla \cdot(\rho \mathbf{v} \otimes \mathbf{v})=0 .
$$

A different derivation can be found in [4]. The system consisting of (3.1) and (3.2) is known as the pressureless Euler system [17]. The idea is that, given a time horizon $T>0$ and two Borel probabilities with finite second moments $\rho_{0}, \rho_{T}$ (such that Monge's problem of optimally transporting $\rho_{0}$ into $\rho_{T}$ has a solution $M)$, the speed-curve $(\rho, \mathbf{v})$ of the geodesic connecting $\rho_{0}$ and $\rho_{T}$ satisfies the pressureless Euler system with given initial and terminal densities.

3.2. Generalized geodesics: A new metric space. Motivated by the lack of convexity of $\mu \rightarrow W_{2}^{2}(\mu, v)$ along geodesics, Ambrosio et al. [3] have constructed generalized geodesics in the Wasserstein space in the following manner: given the 
reference probability measure $v \in \mathcal{P}_{2}^{\text {ac }}\left(\mathbb{R}^{d}\right)$ (absolutely continuous with respect to $\mathcal{L}^{d}$ ) and the corresponding gradients of convex functions $M_{0}, M_{T}$ such that $\rho_{0}=M_{0 \#} \nu$ and $\rho_{T}=M_{T \#} \nu$, we interpolate between $\rho_{0}$ and $\rho_{T}$ by

$$
[0, T] \ni t \rightarrow \rho_{t}=\left[(1-t / T) M_{0}+(t / T) M_{T}\right]_{\#} \nu=: M_{t \#} \nu
$$

We define

$$
d_{v}\left(\rho_{0}, \rho_{T}\right)=\left\|M_{0}-M_{T}\right\|_{L^{2}\left(Q ; \mathbb{R}^{d}\right)} .
$$

To fix the ideas, let us take $v:=\left.\mathcal{L}^{d}\right|_{Q}$, where $Q$ is the open unit cube in $\mathbb{R}^{d}$ centered at the origin.

Theorem 3.1. $\left(\mathcal{P}_{2}\left(\mathbb{R}^{d}\right), d_{v}\right)$ is a Polish space.

Proof. The set $\mathcal{M}_{v}$ consisting of all gradients of convex functions lying in $L^{2}\left(Q ; \mathbb{R}^{d}\right)$ is a closed, convex subset of $L^{2}\left(Q ; \mathbb{R}^{d}\right)$ which is isometrically identical to $\left(\mathcal{P}_{2}\left(\mathbb{R}^{d}\right), d_{v}\right)$. Thus, $d_{v}$ defines a complete metric on $\mathcal{P}_{2}\left(\mathbb{R}^{d}\right)$. Separability comes from the separability of $L^{2}\left(Q ; \mathbb{R}^{d}\right)$.

We define $\operatorname{AC}_{v}^{2}\left(0, T ; \mathcal{P}_{2}\left(\mathbb{R}^{d}\right)\right)$ as the set of all $[0, T] \ni t \rightarrow \rho_{t} \in \mathcal{P}_{2}\left(\mathbb{R}^{d}\right)$ for which there exists $\beta \in L^{2}(0, T)$ such that

$$
d_{v}\left(\rho_{s}, \rho_{t}\right) \leq \int_{S}^{t} \beta(\tau) \mathrm{d} \tau \quad \text { for all } 0 \leq s<t \leq T .
$$

Due to the isometry with $\left(\mathcal{M}_{v},\|\cdot\|_{L^{2}\left(Q ; \mathbb{R}^{d}\right)}\right)$, one readily sees that

$$
\rho \in \operatorname{AC}_{v}^{2}\left(0, T ; \mathcal{P}_{2}\left(\mathbb{R}^{d}\right)\right) \Longleftrightarrow M \in H^{1}\left(0, T ; L^{2}\left(Q ; \mathbb{R}^{d}\right)\right)
$$

and the metric derivative

$$
\left|\rho^{\prime}\right|_{v}(t)=\left\|\dot{M}_{t}\right\|_{L^{2}\left(Q ; \mathbb{R}^{d}\right)} \quad \text { for almost every } t \in(0, T) .
$$

Thus, the length of $t \rightarrow \rho_{t}$ is

$$
\ell(\rho)=\int_{0}^{T}\left\|\dot{M}_{t}\right\|_{L^{2}\left(Q ; \mathbb{R}^{d}\right)} \mathrm{d} t
$$

and this easily shows that (3.3) defines a geodesic connecting $\rho_{0}, \rho_{T}$. We have used the subscripts $v$ to distinguish between these notions in the two different cases given by the quadratic Wasserstein distance $W_{2}$ and the new distance $d_{v}$. The obvious inequality $W_{2} \leq d_{v}$ implies

$$
\operatorname{AC}_{v}^{2}\left(0, T ; \mathcal{P}_{2}\left(\mathbb{R}^{d}\right)\right) \subset \mathrm{AC}^{2}\left(0, T ; \mathcal{P}_{2}\left(\mathbb{R}^{d}\right)\right)
$$


for any $v \in \mathcal{P}_{2}^{\text {ac }}\left(\mathbb{R}^{d}\right)$.

By means of counterexample we prove the last two statements in the proposition below. Also, it is worth mentioning that (i) admits a shorter proof (for the optimal transportation oriented reader), based on the uniqueness of the optimal transference plan (see [3], [17]) between $\mu$ and $\nu$. Indeed, if $W_{2}\left(M_{n_{*}} \nu, M_{\#} v\right) \rightarrow 0$, then the second moments of the measures $\left(\mathbf{i d} \times M_{n}\right)_{\#} v$ are obviously uniformly bounded (they, in fact, converge to the second moment of $\left.(\mathbf{i d} \times M)_{\#} v\right)$; then Remark 5.1.5 [3] shows that $\left\{\left(\mathbf{i d} \times M_{n}\right)_{\#} \nu\right\}_{n}$ is tight, i.e., it admits a subsequence weakly convergent as measures to some $P \in \mathcal{P}\left(\mathbb{R}^{2 d}\right)$. A lower semicontinuity argument reveals that $P$ must be an optimal plan between $\mu$ and $\nu$. By uniqueness ( $v$ is absolutely continuous with respect to the Lebesgue measure), $P=(\mathbf{i d} \times M)_{\#} \nu$ and the convergence of the second moments gives $\left(\mathbf{i d} \times M_{n}\right)_{\#} \nu \rightarrow(\mathbf{i d} \times M)_{\#} \nu$ in $\mathcal{P}_{2}\left(\mathbb{R}^{2 d}\right)$. It follows that $M_{n}$ converges weakly in $L^{2}\left(Q ; \mathbb{R}^{d}\right)$ to $M$ and the convergence of the norms shows that we have strong convergence. However, we prefer to give a more elementary, albeit slightly longer proof.

\section{Proposition 3.2.}

(i) The spaces $\left(\mathcal{P}_{2}\left(\mathbb{R}^{d}\right), W_{2}\right)$ and $\left(\mathcal{P}_{2}\left(\mathbb{R}^{d}\right), d_{v}\right)$ are topologically equivalent.

(ii) If $d>1$, then they are not metrically equivalent.

(iii) Furthermore, if $d>1$ the inclusion (3.6) is strict.

Proof. (i). To prove the first statement note that due to $W_{2} \leq d_{v}$ we only need to show that inside any ball of $\left(\mathcal{P}_{2}\left(\mathbb{R}^{d}\right), W_{2}\right)$ there lies a ball of $\left(\mathcal{P}_{2}\left(\mathbb{R}^{d}\right), d_{v}\right)$. In other words, let us consider $\mu_{n}, \mu \in \mathcal{P}_{2}\left(\mathbb{R}^{d}\right)$ such that $W_{2}\left(\mu_{n}, \mu\right) \rightarrow 0$ as $n \rightarrow \infty$. We want to show that $M_{n} \rightarrow M$ in $L^{2}\left(Q ; \mathbb{R}^{d}\right)$, where $M_{n \#} \nu=\mu_{n}, M_{\#} \nu=\mu$ and $M_{n}, M$ are almost everywhere gradients of convex functions $\Phi_{n}, \Phi$ defined on $\mathbb{R}^{d}$ and restricted to $Q$. We have

$$
\begin{aligned}
& W_{2}\left(\mu_{n}, \mu\right) \rightarrow 0 \Longleftrightarrow \mu_{n} \rightarrow \mu \text { weakly } \star \text { as measures, } \\
& \quad \text { and } \int_{\mathbb{R}^{d}}|x|^{2} \mathrm{~d} \mu_{n} \rightarrow \int_{\mathbb{R}^{d}}|x|^{2} \mathrm{~d} \mu .
\end{aligned}
$$

That is,

$$
\begin{aligned}
& \int_{Q} \varphi \circ M_{n}(x) \mathrm{d} x \rightarrow \int_{Q} \varphi \circ M(x) \mathrm{d} x \\
& \qquad \text { for all } \varphi \in C_{C}\left(\mathbb{R}^{d}\right) \text {, and }\left\|M_{n}\right\| \rightarrow\|M\|,
\end{aligned}
$$

where $\|\cdot\|$ is the standard norm of $L^{2}\left(Q ; \mathbb{R}^{d}\right)$. The restrictions of $\Phi_{n}$ to $\bar{Q}$ are continuous, therefore, bounded on $\bar{Q}$ and we can assume $\Phi_{n}\left(x_{n}\right)=0=\min \Phi_{n}$ over $\bar{Q}$. Thus,

$$
0 \leq \Phi_{n}(x) \leq M_{n} x \cdot\left(x-x_{n}\right) \leq 2\left|M_{n} x\right| \text { for almost every } x \in Q
$$


which by (3.7) leads to the fact that $\left\{\Phi_{n}\right\}_{n}$ is bounded in $L^{2}(Q)$. Furthermore, since the pointwise and so, distributional, gradients $M_{n}$ lie and are uniformly bounded in $L^{2}\left(Q ; \mathbb{R}^{d}\right)$, we infer $\left\{\Phi_{n}\right\}_{n}$ is bounded in $H^{1}(Q)$. Thus, possibly up to a subsequence,

$$
\Phi_{n} \rightarrow \Phi, \quad \text { weakly in } H^{1}(Q) \text { and strongly in } L^{2}(Q) \text {, as } n \rightarrow \infty .
$$

We can also assume $\Phi_{n} \rightarrow \Phi$ almost everywhere in $Q$. We know $\Psi:=\lim \sup \Phi_{n}$ is also convex. Since $\Phi_{n}$ converges almost everywhere to $\Phi$, we infer $\Psi=\Phi$ almost everywhere in $Q$. By the convexity of both, $\Phi \equiv \Psi$ everywhere in $Q$. Thus, we can further extract a subsequence (not relabelled) such that $\Phi_{n} \rightarrow \Phi$ everywhere in $Q$. Now let $A \subset Q$ be the set of full measure where all gradients $M_{n}, \nabla \Phi$ exist and where the pointwise convergence mentioned above holds. Take $Q_{r}:=\left(-\frac{1}{2}+r, \frac{1}{2}-r\right)^{d}$ for sufficiently small $r>0$. Due to

$$
\frac{1}{r}\left[\Phi_{n}\left(x \pm r \mathbf{e}_{i}\right)-\Phi_{n}(x)\right] \geq \pm M_{n} x \cdot \mathbf{e}_{i}
$$

$$
\text { for every } x \in A \cap Q_{r} \text { and any } i=1, \ldots, d \text {, }
$$

we deduce that $\left\{M_{n} x\right\}_{n}$ is bounded for $x \in A \cap Q_{r}$. Any accumulation point must necessarily be $\nabla \Phi(x)$, so the entire sequence $M_{n} x$ must converge to $\nabla \Phi(x)$. Thus, by letting $r \rightarrow 0$, we see that $M_{n}$ converges to $\nabla \Phi$ almost everywhere in $Q$, which implies that $\varphi \circ M_{n}$ converges to $\varphi \circ \nabla \Phi$ almost everywhere in $Q$ for any $\varphi \in C\left(\mathbb{R}^{d}\right)$. Furthermore, by Dominated Convergence, we conclude

$$
\int_{Q} \varphi \circ M_{n}(x) \mathrm{d} x \rightarrow \int_{Q} \varphi \circ \nabla \Phi(x) \mathrm{d} x \quad \text { for all } \varphi \in C_{c}\left(\mathbb{R}^{d}\right),
$$

which by (3.7) yields $\nabla \Phi_{\#} \nu=M_{\#} \nu$. But $M$ is also the restriction to $Q$ of the gradient of a convex function on $\mathbb{R}^{d}$. According to Brenier [5], $M=\nabla \Phi$ almost everywhere in $Q$. Thus, $M_{n} \rightarrow M$ almost everywhere in $Q$. Furthermore, since $\Phi_{n} \rightarrow \Phi$ in $L^{2}(Q)$, the inequality (3.8) shows that $\left|M_{n}\right|$ is bounded over $Q_{r}$ by an $L^{2}\left(Q_{r}\right)$ function for all $r>0$ sufficiently small. We infer $M_{n} \rightarrow M$ as distributions. The convergence of the norms (3.7) finishes the proof of the first statement.

(ii) To prove the other statements let us specialize to the case $d=2$. It will be obvious how to extend the following construction to higher dimensions. Let

$$
\mu_{t}=\frac{1}{2}\left(\delta_{(-\tan t, 1)}+\delta_{(\tan t,-1)}\right) \quad \text { for } t \in[0, \pi / 4]=\bar{I},
$$

where $I:=(0, \pi / 4)$. Then

$$
W_{2}\left(\mu_{t}, \mu_{s}\right)=|\tan t-\tan s| \leq 2|t-s| \quad \text { for all }(t, s) \in I^{2}
$$


which implies

$$
\mu \in \operatorname{AC}^{\infty}\left(I ; \mathcal{P}_{2}\left(\mathbb{R}^{2}\right)\right) \subset \operatorname{AC}^{2}\left(I ; \mathcal{P}_{2}\left(\mathbb{R}^{2}\right)\right) .
$$

On the other hand, the optimal map pushing $v$ forward to $\mu_{t}$ is given by

$$
M_{t} x= \begin{cases}(-\tan t, 1) & \text { if } x \in D_{t}^{+}, \\ (\tan t,-1) & \text { if } x \in D_{t}^{-},\end{cases}
$$

where $D_{t}^{ \pm}$are the two congruent trapezoids obtained by cutting the square $Q$ by the line passing through the origin and orthogonal to the segment connecting the two points in the support of $\mu_{t}$. Consequently, we can compute (assume $t>s$ )

$$
d_{v}^{2}\left(\mu_{t}, \mu_{s}\right)=(\tan t-\tan s)(4 \tan t \tan s+\tan t-\tan s+4) .
$$

We infer that

$$
\frac{d_{v}\left(\mu_{t}, \mu_{s}\right)}{W_{2}\left(\mu_{t}, \mu_{s}\right)}=\sqrt{1+4 \cot (t-s)} \rightarrow \infty \quad \text { as } s \rightarrow t^{-}, \text {for all } t \in I .
$$

First of all, this shows that, indeed, the metrics $W_{2}$ and $d_{v}$ are not equivalent.

(iii) Secondly,

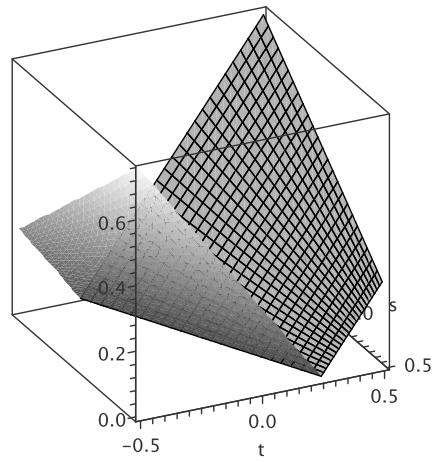

FIGURE 3.1. Graph (parametric in $t, s$ ) of $\Phi_{t_{0}}, t_{0}=\arctan 0.5$ assume $\mu \in \operatorname{AC}_{v}^{2}\left(I ; \mathcal{P}_{2}\left(\mathbb{R}^{2}\right)\right)$. Then there exists $\beta \in L^{2}(I)$ such that (3.5) holds for the path $\mu$. We see that

$$
\lim _{s \rightarrow t} \frac{W_{2}\left(\mu_{s}, \mu_{t}\right)}{|s-t|}=\sec ^{2} t .
$$

for all $t \in I$. Thus, if $t$ is also a Lebesgue point for $\beta$, we deduce $\lim _{s \rightarrow t^{-}} \frac{\int_{s}^{t} \beta(\tau) \mathrm{d} \tau}{W_{2}\left(\mu_{t}, \mu_{s}\right)}=\beta(t) \cos ^{2} t<\infty$

for almost every $t \in I$. 
In view of (3.5), this contradicts (3.10). Thus, the path $\mu$ lies in

$$
\operatorname{AC}^{2}\left(I ; \mathcal{P}_{2}\left(\mathbb{R}^{2}\right)\right) \backslash \mathrm{AC}_{v}^{2}\left(I ; \mathcal{P}_{2}\left(\mathbb{R}^{2}\right)\right) .
$$

In particular, (iii) is proved.

Theorem 8.3.1 [3] proves the existence of a minimal-norm Borel velocity field $\mathbf{w}:(0, T) \times \mathbb{R}^{d} \rightarrow \mathbb{R}^{d}$ such that the continuity equation (3.1) is satisfied by the pair $(\rho, \mathbf{w})$. Furthermore, among all Borel fields $\mathbf{u}$ satisfying (3.1) and $\mathbf{u}_{t} \in$ $L^{2}\left(\rho_{t} ; \mathbb{R}^{d}\right)$ for almost every $t \in(0, T), \mathbf{w}$ is the only one with minimal $L^{2}$-norm for almost every $t \in(0, T)$. It also satisfies $\left\|\mathbf{w}_{t}\right\|_{L^{2}\left(\rho_{t} ; \mathbb{R}^{d}\right)}=\left|\rho^{\prime}\right|(t)$ for almost every $t \in(0, T)$. The natural question now is whether there exists a Borel velocity field $\mathbf{v}$ satisfying (3.1) and

$$
\|\mathbf{v}\|_{L^{2}\left(\rho_{t} ; \mathbb{R}^{d}\right)}=\left|\rho^{\prime}\right|_{\nu}(t) \quad \text { for almost every } t \in(0, T) .
$$

According to Corollary 2.3, the following is true. Indeed, the proof consists of the same argument used in the beginning of this section to prove (3.1). The only difference is that now the optimal maps $M_{t}$ are not the ones pushing $\mu_{0}$ forward to $\mu_{t}$, but the ones pushing $v$ forward to $\mu_{t}$.

Proposition 3.3. Let $\rho \in \mathrm{AC}_{v}^{2}\left(0, T ; \mathcal{P}_{2}\left(\mathbb{R}^{d}\right)\right)$ be such that the family of corresponding optimal maps $M_{t}$ (i.e., gradients of convex functions such that $\rho_{t}=M_{t \#} \nu$ ) satisfies condition $(\mathrm{H})$. Then there exists a Borel map $\mathbf{v}:(0, T) \times \mathbb{R}^{d} \rightarrow \mathbb{R}^{d}$ such that (3.1) and (3.11) hold.

Remark 3.4. Note that $(\mathrm{H})$ is satisfied if all $\mu_{t}$ are absolutely continuous with respect to the Lebesgue measure (in which case the maps $M_{t}$ are invertible) or are fully supported at discrete points (convex, possibly countable, combinations of Dirac masses).

To return to the generalized geodesic given by (3.3), we are now ready to prove the following result.

Proposition 3.5. Let $\rho_{0}, \rho_{T} \in \mathcal{P}_{2}\left(\mathbb{R}^{d}\right)$ be given. Then there exists a Borel velocity $\mathbf{v}:(0, T) \times \mathbb{R}^{d} \rightarrow \mathbb{R}^{d}$ associated with the $d_{v}$-geodesic given by (3.3), i.e., satisfying (3.1) and (3.11) for this geodesic. Furthermore, (3.2) is also satisfied.

Proof. According to Corollary 2.6, (Eq) is satisfied. Thus, Corollary 2.3 applies to give us the required velocity field. To obtain (3.2) for the present $(\rho, \mathbf{v})$ pair we repeat the argument used in the beginning of this section to obtain the same equation for the $W_{2}$-geodesic. That is, we start with $\ddot{M}_{t} \equiv 0$, then multiply by $\phi\left(t, M_{t} x\right)$ and integrate by parts on $(0, T) \times Q$ to obtain (3.2) via (2.5).

3.3. Repulsivelattractive Euler-Monge-Ampère systems with uniform background. In this subsection we look at special variational solutions for EulerMonge-Ampère systems: solutions lying in $\operatorname{AC}_{v}^{2}\left(0, T ; \mathcal{P}_{2}\left(\mathbb{R}^{d}\right)\right)$. We would like to point out that even though our conclusions may be regarded as applications of 
Corollary 2.3, they do, in fact, correspond to the "invertible case" in which the maps $M_{t}$ are essentially injective. Thus, the velocity $\mathbf{v}_{t}$ is well defined as $\dot{M}_{t} \circ M_{t}^{-1}$.

We consider the system introduced by Brenier and Loeper [7] as an asymptotic approximation to the repulsive Euler-Poisson system, i.e.,

$$
\left\{\begin{array}{l}
\partial_{t} \rho+\nabla_{x} \cdot(\rho \mathbf{v})=0, \\
\partial_{t}(\rho \mathbf{v})+\nabla_{x} \cdot(\rho \mathbf{v} \otimes \mathbf{v})=-\rho \nabla_{x} \Phi \quad \text { in }(0, T) \times \mathbb{R}^{d}, \\
\left(\mathbf{i d}-\nabla_{x} \Phi_{t}\right)_{\#} \rho_{t}=v, \\
x \mapsto \frac{|x|^{2}}{2}-\Phi_{t}(x) \quad \text { is convex for } t \in(0, T) .
\end{array}\right.
$$

To allow for solutions consisting of Borel probability measures which are not necessarily absolutely continuous with respect to the Lebesgue measure, the author and his collaborators [11] introduced an accordingly modified version of the system:

$$
\left\{\begin{array}{l}
\partial_{t} \rho+\nabla_{x} \cdot(\rho \mathbf{v})=0, \\
\partial_{t}(\rho \mathbf{v})+\nabla_{x} \cdot(\rho \mathbf{v} \otimes \mathbf{v})=\rho[\bar{\gamma}-\mathbf{i d}] \quad \text { in }(0, T) \times \mathbb{R}^{d}
\end{array}\right.
$$

In general, the barycentric projection $\bar{\gamma}_{\mu}: \mathbb{R}^{d} \rightarrow \mathbb{R}^{d}$ of a plan $\gamma \in \mathcal{P}\left(\mathbb{R}^{d} \times \mathbb{R}^{d}\right)$ onto its second marginal $\mu:=\pi_{\#}^{2} \gamma$ is uniquely defined [3] $\mu$-almost everywhere by

$$
\bar{\gamma}_{\mu}(y):=\int_{\mathbb{R}^{d}} x \mathrm{~d} \gamma_{y}(x) \text { for } \mu \text {-almost every } y \in \mathbb{R}^{d},
$$

where $\gamma$ is disintegrated as

$$
\gamma=\int_{\mathbb{R}^{d}} \gamma y \mathrm{~d} \mu(y) .
$$

In the right hand side of the second equation in (3.13) we consider $\bar{\gamma}$ at time $t$ to be the barycentric projection onto $\rho_{t}$ of the unique optimal coupling

$$
\gamma_{t}:=\left(\mathbf{i d} \times M_{t}\right)_{\#} \nu \in \Gamma_{o}\left(\nu, \rho_{t}\right),
$$

where $M_{t}$ is the optimal map pushing $v$ forward to $\rho_{t}$. Note that when there exists an optimal map $\nabla_{x} \Psi_{t}$ such that $\nabla_{x} \Psi_{t \#} \rho_{t}=v$ (say, when $\rho_{t}$ are all absolutely continuous with respect to the Lebesgue measure), then (3.13) reverts back to (3.12) with $\Phi_{t}(x)=|x|^{2} / 2-\Psi_{t}(x)$.

In this paragraph we briefly describe the approach in [11], where it was proved that the critical paths of a certain action functional on $\mathrm{AC}^{2}\left(0, T ; \mathcal{P}_{2}\left(\mathbb{R}^{d}\right)\right)$ are 
solutions for (3.13). The action

$$
\mathcal{A}_{T}(\sigma):=\frac{1}{2} \int_{0}^{T}\left[\left|\sigma^{\prime}\right|(t)^{2}-W_{2}^{2}\left(\sigma_{t}, v\right)\right] \mathrm{d} t
$$

was considered over the set of all paths in $\operatorname{AC}^{2}\left(0, T ; \mathcal{P}_{2}\left(\mathbb{R}^{d}\right)\right)$ with fixed endpoints. It was proved that a minimizer of $\mathcal{A}_{T}$ over this set is a solution for (3.13). The proof was Eulerian in nature: we fixed $\sigma \in \mathrm{AC}^{2}\left(0, T ; \mathcal{P}_{2}\left(\mathbb{R}^{d}\right)\right)$ and $\xi \in C_{c}^{\infty}\left((0, T) \times \mathbb{R}^{d} ; \mathbb{R}^{d}\right)$, then defined $\sigma_{t}^{s}=(\mathbf{i d}+s \xi(t, \cdot))_{\#} \sigma_{t}$ which was used as an admissible variation to establish the assertion. Thus, even though minimizers were not obtained unless $d=1$, it was shown that such minimizers (if they existed) would be solutions for the two-point boundary problem. We should point out that the velocity $\mathbf{v}$ of the minimizing path is the minimal-norm velocity, i.e., the one satisfying

$$
\left\|\mathbf{v}_{t}\right\|_{L^{2}\left(\sigma_{t} ; \mathbb{R}^{d}\right)}=\left|\sigma^{\prime}\right|(t)=\inf \left\|\mathbf{w}_{t}\right\|_{L^{2}\left(\sigma_{t} ; \mathbb{R}^{d}\right)},
$$

where the infimum is taken along all Borel velocities $\mathbf{w}$ (i.e., satisfying (3.1)) such that $\mathbf{w}_{t} \in L^{2}\left(\sigma_{t} ; \mathbb{R}^{d}\right)$ for almost every $t \in(0, T)$. Since in the case $d=1$ the problem of existence of minimizers was dealt with by switching to a Lagrangian formulation of the problem in terms of the optimal maps $M_{t}$, we now address the question whether this can also be done in higher dimensions. At the outset, this is not at all obvious: indeed, only in one-dimension is it generally true that $\left|\sigma^{\prime}\right|(t)=\left\|\dot{M}_{t}\right\|_{L^{2}(Q)}$. Thus, only then is it true that

$$
\mathcal{A}_{T}(\sigma)=\frac{1}{2}\left(\|\dot{M}\|^{2}-\left\|M-\mathbf{i d}_{Q}\right\|^{2}\right)=: \mathcal{F}(M),
$$

where the norm $\|\cdot\|$ represents the standard $L^{2}((0, T) \times Q)$-norm. This opens the possibility of looking for a solution pair $(\sigma, \mathbf{v})$ with $\sigma \in \operatorname{AC}_{v}^{2}\left(0, T ; \mathcal{P}_{2}\left(\mathbb{R}^{d}\right)\right)$ and $\mathbf{v}$ the corresponding velocity field, since we know that in this case

$$
\left|\sigma^{\prime}\right|_{\nu}(t)=\left\|\mathbf{v}_{t}\right\|_{L^{2}\left(\sigma_{t} ; \mathbb{R}^{d}\right)}=\left\|\dot{M}_{t}\right\|_{L^{2}\left(\sigma_{t} ; \mathbb{R}^{d}\right)} .
$$

Let $M_{0}, \quad M_{T} \in L^{2}\left(Q ; \mathbb{R}^{d}\right)$ be the restrictions to $Q$ of two gradients of convex functions over $\mathbb{R}^{d}$. Denote by $\mathcal{H}$ the set of all paths in $H^{1}\left(0, T ; L^{2}\left(Q ; \mathbb{R}^{d}\right)\right)$ such that $M_{t}$ is a gradient of a convex function for all $t \in[0, T]$. We shall next prove the following result.

Proposition 3.6. If $0<T<\pi$, then there exists a unique minimizer $M$ for $\mathcal{F}$ over $\mathcal{H}$ with fixed endpoints $M_{0}, M_{T}$, respectively.

Proof. We consider the continuous bilinear form defined by

$$
\mathcal{B}(M, N)=\int_{0}^{T}\left(\langle\dot{M}, \dot{N}\rangle_{Q}-\langle M, N\rangle_{Q}\right) \mathrm{d} t
$$

$$
\text { for } M, N \in H^{1}\left(0, T ; L^{2}\left(Q ; \mathbb{R}^{d}\right)\right),
$$


where $\langle\cdot, \cdot\rangle_{Q}$ denotes the standard inner product in $L^{2}\left(Q ; \mathbb{R}^{d}\right)$. Minimizing $\mathcal{F}$ over paths with prescribed endpoints $M_{0}, M_{T}$ is equivalent to minimizing $\mathcal{B}(M, M) / 2$ over paths with endpoints $N_{0}:=M_{0}-\mathbf{i d} \mathbf{d}_{Q}$ and $N_{T}:=M_{T}-\mathbf{i d} \mathbf{d}_{Q}$. Consider the linear interpolation $N_{t}:=(1-t / T) N_{0}+(t / T) N_{T}$ and note that we arrive to minimizing

$$
\frac{1}{2} B(S, S)-\int_{0}^{T}\left\langle S_{t}, N_{t}\right\rangle_{Q} \mathrm{~d} t
$$

over $S \in H_{0}^{1}\left(0, T ; L^{2}\left(Q ; \mathbb{R}^{d}\right)\right)$. We apply Poincarés inequality from [11, Proposition 2 , with $\left.s_{0}=0\right]$ to $S=L^{2}\left(Q ; \mathbb{R}^{d}\right)$ and obtain

$$
\mathcal{B}(S, S) \geq\left(1-\frac{T^{2}}{\pi^{2}}\right)\|\dot{S}\|^{2} \geq \frac{\pi^{2}}{T^{2}}\left(1-\frac{T^{2}}{\pi^{2}}\right)\|S\|^{2}
$$

for all $S \in H_{0}^{1}\left(0, T ; L^{2}\left(Q ; \mathbb{R}^{d}\right)\right)$. Existence and uniqueness follow by LionsStampacchia's Theorem [12].

Remark 3.7. The problem with using this result to obtain weak solutions for (3.13) is that, since we are minimizing over a closed, convex subset of the space $H^{1}\left(0, T ; L^{2}\left(Q ; \mathbb{R}^{d}\right)\right)$, we are not able to obtain an Euler-Lagrange equation for the minimizer. Indeed, we can only infer that $M$ is the unique path in

$$
C\left(M_{0}, M_{T}\right):=\left\{N \in H^{1}\left(0, T ; L^{2}\left(Q ; \mathbb{R}^{d}\right)\right) \mid N_{0}=M_{0}, N_{T}=M_{T}\right\}
$$

for which the following variational inequality [12] holds

$$
\int_{0}^{T}\left\{\left\langle\dot{M}_{t}, \dot{M}_{t}-\dot{N}_{t}\right\rangle_{Q}+\left\langle\mathbf{i d}_{Q}-M_{t}, M_{t}-N_{t}\right\rangle_{Q}\right\} \mathrm{d} t \leq 0 \quad \text { for all } N \in C\left(M_{0}, M_{T}\right) .
$$

We have not been able to employ this to prove that $M$ produces a distributional solution for (3.13).

We next show that, under certain conditions, the minimizer obtained above is a weak solution for the two-point boundary problem associated with (3.12).

Theorem 3.8. Let $0<T<\pi / 2$ and $\rho_{0}, \rho_{T} \in \mathcal{P}_{2}\left(\mathbb{R}^{d}\right)$ be such that at least one of the optimal maps such that $M_{s \#} \nu=\rho_{s}$ for $s=0, T$ satisfies $\nabla M_{s} \geq I_{d}$. Then the minimizer from Proposition 3.6 is a distributional solution for (3.12). Furthermore, the probabilities $\rho_{t}$ are absolutely continuous with respect to the Lebesgue measure for all $t \in[0, T]$ except, possibly, one of the endpoints.

Proof. The variational inequality from Remark 3.7 is satisfied if

$$
M \in H^{2}\left(0, T ; L^{2}\left(Q ; \mathbb{R}^{d}\right)\right)
$$

and

$$
\ddot{M}_{t} x+M_{t} x=x \quad \text { in }(0, T) \times Q .
$$


Since the endpoints $M_{0}, M_{T}$ are prescribed, we obtain an explicit expression for the solution

$$
M_{t} x=\left(M_{0} x-x\right)\left(\cos t-\frac{\cos T}{\sin T} \sin t\right)+\left(M_{T} x-x\right) \frac{\sin t}{\sin T}+x .
$$

Assume, without loss of generality, that $\nabla M_{0} \geq I_{d}$, i.e., $M_{0}-\mathbf{i d} \mathbf{d}_{Q}$ is the gradient of a convex function, say $\Psi$. Then $M_{t}$ can be rewritten as

$$
M_{t}=\left(\cos t-\frac{\cos T}{\sin T} \sin t\right) \nabla \Psi+\frac{\sin t}{\sin T} M_{T}+\left(1-\frac{\sin t}{\sin T}\right) \mathbf{i d}_{Q},
$$

which is a gradient of a convex function for all $t \in[0, T]$. Due to the uniqueness of $M \in C\left(M_{0}, M_{T}\right)$ satisfying the inequality from Remark 3.7, we deduce that $M$ in the above expression is the unique minimizer given by Proposition 3.6. Note that $M_{t}$ is invertible for all $t \in[0, T)$ and $\mathbf{v}_{t}=\dot{M}_{t} \circ M_{t}^{-1}$. We also note that $\rho_{t}$ is absolutely continuous with respect to the Lebesgue measure for $t \in[0, T)$ and place $\nabla \Phi_{t}:=\mathbf{i d}_{Q}-M_{t}^{-1}$ (we know $M_{t}^{-1}$ is also the gradient of a convex function, so such $\Phi_{t}$ exists). Take an arbitrary test function $\xi \in C_{c}^{\infty}\left((0, T) \times \mathbb{R}^{d}\right)$ and multiply (3.18) by $\xi\left(t, M_{t} x\right)$. We then write

$$
\int_{0}^{T} \int_{Q} \ddot{M}_{t} x \xi\left(t, M_{t} x\right) \mathrm{d} x \mathrm{~d} t=\int_{0}^{T} \int_{Q}\left(x-M_{t} x\right) \xi\left(t, M_{t} x\right) \mathrm{d} x \mathrm{~d} t .
$$

Next we integrate by parts once with respect to time in the left hand side, use the facts that $\dot{M}_{t}=\mathbf{v}_{t} \circ M_{t}, \mathbf{i} \mathbf{d}_{Q}-\nabla \Phi_{t}=M_{t}^{-1}$ and $M_{t \#} \nu=\rho_{t}$ to conclude.

The attractive case features a different sign in the right hand side of the momentum equation:

$$
\left\{\begin{array}{l}
\partial_{t} \rho+\nabla_{x} \cdot(\rho \mathbf{v})=0, \\
\partial_{t}(\rho \mathbf{v})+\nabla_{x} \cdot(\rho \mathbf{v} \otimes \mathbf{v})=\rho \nabla_{x} \Phi \quad \text { in }(0, T) \times \mathbb{R}^{d}, \\
\left(\mathbf{i d}-\nabla_{x} \Phi_{t}\right)_{\#} \rho_{t}=v, \\
x \mapsto \frac{|x|^{2}}{2}-\Phi_{t}(x) \quad \text { is convex for } t \in(0, T) .
\end{array}\right.
$$

The difference from the repulsive case is that the Lagrangian now becomes

$$
\mathcal{U}_{T}(\sigma):=\frac{1}{2} \int_{0}^{T}\left[\left|\sigma^{\prime}\right|(t)^{2}+W_{2}^{2}\left(\sigma_{t}, v\right)\right] \mathrm{d} t
$$

Just as in [11], one can prove that a minimizer of $\mathcal{U}_{T}$ satisfies a weak form of (3.19), namely (3.13) except that the right hand side of the momentum equation has a changed sign. 
As before, we'd like to investigate the existence issue in $\mathrm{AC}_{v}^{2}\left(0, T ; \mathcal{P}_{2}\left(\mathbb{R}^{d}\right)\right)$ instead of $\operatorname{AC}^{2}\left(0, T ; \mathcal{P}_{2}\left(\mathbb{R}^{d}\right)\right)$. Likewise, by identifying the measures $\sigma_{t}$ with the corresponding optimal maps $M_{t}$ such that $M_{t \#} \nu=\sigma_{t}$, we obtain

$$
\mathcal{U}_{T}(\sigma)=\frac{1}{2}\left(\|\dot{M}\|^{2}+\left\|M-\mathbf{i d}_{Q}\right\|^{2}\right)=: G(M) \quad \text { for all } \sigma \in \mathrm{AC}_{v}^{2}\left(0, T ; \mathcal{P}_{2}\left(\mathbb{R}^{d}\right)\right)
$$

Much more can be said of (3.19) than of (3.12).

Theorem 3.9. For any $T>0$ and any $\rho_{0}, \rho_{T} \in \mathcal{P}_{2}\left(\mathbb{R}^{d}\right)$ there exists a unique minimizer for $G$ over $C\left(M_{0}, M_{T}\right)$. The probabilities $\rho_{t}$ are absolutely continuous with respect to $\mathcal{L}^{d}$ for all $t \in(0, T)$ and the pair $(\rho, \mathbf{v})$ is a distributional solution for (3.19), where $\mathbf{v}$ is the velocity associated with $\rho$ in $\mathrm{AC}_{v}^{2}$.

Proof. For existence and uniqueness we return to the proof of Proposition 3.6 and note that the only reason why the restriction $T<\pi$ was needed came from the Poincarés inequality. This inequality was necessary to ensure the coercivity of the quadratic form $S \rightarrow \mathcal{B}(S, S)$ applied to paths lying in $H_{0}^{1}\left(0, T ; L^{2}\left(Q ; \mathbb{R}^{d}\right)\right)$. Note that in the present case, given the bilinear form

$$
\begin{aligned}
\mathcal{E}(M, N)=\frac{1}{2} \int_{0}^{T}\left(\langle\dot{M}, \dot{N}\rangle_{Q}+\langle M, N\rangle_{Q}\right) \mathrm{d} t & \\
& \text { for } M, N \in H^{1}\left(0, T ; L^{2}\left(Q ; \mathbb{R}^{d}\right)\right),
\end{aligned}
$$

we see that its associated quadratic form is automatically coercive, regardless of $T$. The rest of the existence proof goes the same way. Furthermore, we observe that the variational inequality corresponding to this unique minimizer is similar to the one spelled out in Remark 3.7, except that the term $\mathbf{i d}_{Q}-M_{t}$ is replaced by $M_{t}-\mathbf{i d}_{Q}$. Fortunately, the unconstrained solution of $\ddot{M}-M+\mathbf{i} \mathbf{d}_{Q}=0$ over $H^{1}\left(0, T ; L^{2}\left(Q ; \mathbb{R}^{d}\right)\right)$ with fixed endpoints $M_{0}, M_{T}$ is given by

$$
M_{t}=\left(1-\frac{\sinh t+\sinh (T-t)}{\sinh T}\right) \mathbf{i d}_{Q}+\frac{\sinh (T-t)}{\sinh T} M_{0}+\frac{\sinh t}{\sinh T} M_{T},
$$

which is clearly the gradient of a convex function for all $t \in[0, T]$. Thus, $\rho_{t}:=M_{t \#} \nu$ must be the above found minimizer. In fact, $M_{t}$ is the gradient of a uniformly convex function except, possibly, when $t=0, T$. Thus, $\rho_{t} \ll \mathcal{L}^{d}$ and $M_{t}$ is invertible and satisfies $M_{t \#}^{-1} \rho_{t}=v$ for all $t \in(0, T)$. Upon placing $\mathbf{v}_{t}:=\dot{M}_{t} \circ M_{t}^{-1}$, we conclude our present proof by following the proof of Theorem 3.8 . 
3.4. Monokinetic solutions for the nonlinear Vlasov system. In this subsection we discuss possible applications to the nonlinear Vlasov system

$$
\left\{\begin{array}{l}
\partial_{t} f+v \cdot \nabla_{x} f=\nabla_{v} \cdot\left(f \nabla_{x} \Phi\right), \\
\Phi_{t}(x)=\int_{\mathbb{R}^{d}} W(x-y) \rho_{t}(y) \mathrm{d} y \quad \text { in }(0, T) \times \mathbb{R}^{d} \times \mathbb{R}^{d}, \\
\rho_{t}(x)=\int_{\mathbb{R}^{d}} f_{t}(x, v) \mathrm{d} v,
\end{array}\right.
$$

where $W$ is a smooth potential $W: \mathbb{R}^{d} \rightarrow \mathbb{R}$. Assume the initial data is in the set of probabilities on $\mathbb{R}^{2 d}$ such that $f_{0}(x, v)=\left(M_{0}, N_{0}\right)_{\#} v$ (again, $v$ denotes the $d$-dimensional Lebesgue measure restricted to the unit cube $Q$ ) and $M_{0}, N_{0} \in$ $L^{2}\left(Q ; \mathbb{R}^{d}\right)$. This means

$$
\iint_{\mathbb{R}^{2}} \varphi(x, v) \mathrm{d} f_{0}(x, v)=\int_{Q} \varphi\left(M_{0} y, N_{0} y\right) \mathrm{d} y \quad \text { for all } \varphi \in C_{C}\left(\mathbb{R}^{2 d}\right) .
$$

Let us introduce the initial value problem

$$
\ddot{M}_{t} y=-\int_{Q} \nabla W\left(M_{t} y-M_{t} z\right) \mathrm{d} z,\left.\quad M\right|_{t=0}=M_{0},\left.\dot{M}\right|_{t=0}=N_{0} .
$$

We may rewrite (3.23) as a first-order system and use the Cauchy-LipschitzPicard Theorem [8] to prove that, if $W \in C^{1,1}\left(\mathbb{R}^{d}\right)$, then for any initial data $\left(M_{0}, N_{0}\right) \in L^{2}\left(Q ; \mathbb{R}^{d}\right) \times L^{2}\left(Q ; \mathbb{R}^{d}\right)$ the problem (3.23) admits a unique solution

$$
M \in H^{2}\left(0, \infty ; L^{2}\left(Q ; \mathbb{R}^{d}\right)\right) .
$$

We can then easily check that

$$
f(t, \cdot, \cdot):=\left(M_{t}, \dot{M}_{t}\right)_{\#} \nu \quad \text { with } f_{0}(x, v)=\left(M_{0}, N_{0}\right)_{\#} \nu
$$

satisfies (3.22) in the sense of distributions.

By monokinetic solutions we understand distributional solutions of the form

$$
f_{t}(x, v)=\rho_{t}(x) \delta_{\mathbf{u}_{t}(x)}(v) .
$$

The pair $(\rho, \mathbf{u})$ would satisfy

$$
\left\{\begin{array}{l}
\partial_{t} \rho+\nabla_{x} \cdot(\rho \mathbf{u})=0, \\
\partial_{t}(\rho \mathbf{u})+\nabla_{x} \cdot(\rho \mathbf{u} \otimes \mathbf{u})=\rho \nabla_{x} \Phi \quad \text { in }(0, T) \times \mathbb{R}^{d}, \\
\Phi_{t}(x)=\int_{\mathbb{R}^{d}} W(x-y) \rho_{t}(y) \mathrm{d} y,
\end{array}\right.
$$


in the sense of distributions. Of course, if one could find a solution for (3.23) consisting of gradients of convex functions and satisfying (Eq), then the velocity field $\mathbf{u}$ given by Corollary 2.3 would pair up with $\rho_{t}=M_{t \#} \nu$ to yield a distributional solution for (3.24). We have not been able to establish a general result in this direction. However, we provide an example below.

Example. Let $W(y)=|y|^{2} / 2$ be the potential. Note that in this case the $\mathrm{ODE}$ in (3.23) becomes linear and reads

$$
\ddot{M}_{t} y+M_{t} y=\int_{Q} M_{t} z \mathrm{~d} z .
$$

If $N \in L^{1}\left(Q ; \mathbb{R}^{d}\right)$, let $\bar{N}$ denote the average of $N$ over $Q$. In order to solve the associated two-point boundary problem over $[0, T]$ we integrate first over $Q$ with respect to $y$ to get that the average of $M_{t}$ is a linear function of time. By taking into account the boundary values, we get

$$
\int_{Q} M_{t} y \mathrm{~d} y=\left(1-\frac{t}{T}\right) \bar{M}_{0}+\frac{t}{T} \bar{M}_{T},
$$

so now we can solve (3.25) completely to find

$$
M_{t}=\left(M_{0}-\bar{M}_{0}\right) \frac{\sin (T-t)}{\sin T}+\left(M_{T}-\bar{M}_{T}\right) \frac{\sin t}{\sin T}+\left(1-\frac{t}{T}\right) \bar{M}_{0}+\frac{t}{T} \bar{M}_{T} .
$$

Clearly, if $M_{0}, M_{T} \in L^{2}\left(Q ; \mathbb{R}^{d}\right)$ are gradients of convex functions, then for any $T \in(0, \pi)$ we can apply Corollary 2.3 via Proposition 2.5 to deduce that (3.24) admits a solution for any given $\rho_{0}, \rho_{T} \in \mathcal{P}_{2}\left(\mathbb{R}^{d}\right)$. Note that the choice $W(y)=$ $-|y|^{2} / 2$ leads to a similar expression for $M_{t}$ in which sin is replaced by $\sinh$. In this case the solution for the two-point boundary problem exists (and is given by the corresponding expression) for all time.

Acknowledgements. AT gratefully acknowledges the support provided by the Department of Mathematics of the University of Wisconsin.

We would like to thank M. Feldman, T. Nguyen and W. Gangbo for their valuable comments and suggestions. Last but not least we thank the anonymous referee for pointing out weaknesses of an earlier version and for suggesting major improvements.

\section{REFERENCES}

[1] L. Ambrosio, Transport equation and Cauchy problem for BV vector fields, Invent. Math. 158 (2004), no. 2, 227-260.

http://dx.doi.org/10.1007/s00222-004-0367-2. MR2096794 (2005f:35127)

[2] L. AMBROSIO and W. GANGBO, Hamiltonian ODEs in the Wasserstein space of probability measures, Comm. Pure Appl. Math. 61 (2008), no. 1, 18-53.

http://dx.doi.org/10.1002/cpa.20188. MR2361303 (2009b:37101) 
[3] L. Ambrosio, N. Gigli, and G. Savaré, Gradient Flows in Metric Spaces and in the Space of Probability Measures, Lectures in Mathematics ETH Zürich, Birkhäuser Verlag, Basel, 2005. MR2129498 (2006k:49001)

[4] J.-D. Benamou and Y. Brenier, A computational fluid mechanics solution to the MongeKantorovich mass transfer problem, Numer. Math. 84 (2000), no. 3, 375-393. http://dx.doi.org/10.1007/s002110050002. MR1738163 (2000m:65111)

[5] Y. BRENIER, Polar factorization and monotone rearrangement of vector-valued functions, Comm. Pure Appl. Math. 44 (1991), no. 4, 375-417. http://dx.doi.org/10.1002/cpa.3160440402. MR1100809 (92d:46088)

[6] Y. BRENIER and E. GRENIER, Sticky particles and scalar conservation laws, SIAM J. Numer. Anal. 35 (1998), no. 6, 2317-2328 (electronic). http://dx.doi.org/10.1137/S0036142997317353. MR1655848 (99j:35129)

[7] Y. BRENIER and G. LOEPER, A geometric approximation to the Euler equations: the Vlasov-Monge-Ampere system, Geom. Funct. Anal. 14 (2004), no. 6, 1182-1218. http://dx.doi.org/10.1007/s00039-004-0488-1. MR2135164 (2006a:35259)

[8] H. BREZIS, Analyse fonctionnelle, Collection Mathématiques Appliquées pour la Maitrise. [Collection of Applied Mathematics for the Master's Degree], Masson, Paris, 1983 (French). Théorie et applications. [Theory and applications]. MR697382 (85a:46001)

[9] G. CRIPPA, The flow associated to weakly differentiable vector fields, PhD Thesis, 2007.

[10] R.J. DiPERNA and P.-L. LIONS, Ordinary differential equations, transport theory and Sobolev spaces, Invent. Math. 98 (1989), no. 3, 511-547. http://dx.doi.org/10.1007/BF01393835. MR1022305 (90j:34004)

[11] W. Gangbo, T. NGuYen, and A. Tudorascu, Euler-Poisson systems as action-minimizing paths in the Wasserstein space, Arch. Ration. Mech. Anal. 192 (2009), no. 3, 419-452. http://dx.doi.org/10.1007/s00205-008-0148-y. MR2505360 (2010f:37117)

[12] D. Kinderlehrer and G. StAmpacchia, An Introduction to Variational Inequalities and Their Applications, Classics in Applied Mathematics, vol. 31, Society for Industrial and Applied Mathematics (SIAM), Philadelphia, PA, 2000. Reprint of the 1980 original. MR1786735 (2002d:49001)

[13] R.J. MCCANN, A Convexity Theory for Interacting Gases and Equilibrium Crystals, ProQuest LLC, Ann Arbor, MI, 1994. Thesis (Ph.D.)-Princeton University. MR2636543

[14] T. NGuYen and A. Tudorascu, Pressureless Euler/Euler-Poisson systems via adhesion dynamics and scalar conservation laws, SIAM J. Math. Anal. 40 (2008), no. 2, 754-775. http://dx.doi.org/10.1137/070704459. MR2438785 (2009m:35393)

[15] F. OTTO, Dynamics of labyrinthine pattern formation in magnetic fluids: a mean-field theory, Arch. Rational Mech. Anal. 141 (1998), no. 1, 63-103. http://dx.doi.org/10.1007/s002050050073. MR1613500 (2000j:76145)

[16] A. TUdorascu, Wasserstein kernels for one-dimensional diffusion problems, Nonlinear Anal. 67 (2007), no. 9, 2553-2572. http://dx.doi.org/10.1016/j.na.2006.08.048. MR2343987 (2008f:35211)

[17] C. Villani, Topics in Optimal Transportation, Graduate Studies in Mathematics, vol. 58, American Mathematical Society, Providence, RI, 2003. MR1964483 (2004e:90003)

Department of Mathematics

West Virginia University

Morgantown, WV 26506, U.S.A.

E-MAIL: adriant@math.wvu.edu 
KEY WORDS AND PHRASES: flows of maps, cyclically monotone maps, optimal mass transport, Wasserstein metric, optimal maps, velocities of absolutely continuous curves.

2000 Mathematics Subject Classification: 35M10, 49J40, 82C99.

Received: February 12th, 2009.

Article electronically published on November 5th, 2010. 